\title{
Technical Analysis of Countermeasures against Attack on XML Encryption - or - Just Another Motivation for Authenticated Encryption
}

\author{
Juraj Somorovsky and Jörg Schwenk \\ Horst Görtz Institute for IT Security \\ Ruhr-University Bochum \\ \{juraj.somorovsky, joerg.schwenk\}@rub.de
}

\begin{abstract}
At CCS'11 a new chosen-ciphertext attack on XML Encryption [12] has been presented. This attack is of high relevance, since it allows to decrypt arbitrary encrypted XML payload by issuing 14 server requests per byte on average.

In this paper we discuss several countermeasures against this attack, which have been considered by different framework developers for different scenarios. We analyze the scenarios and show why these countermeasures do not work. Thereby, we motivate for application of authenticated encryption in the XML Encryption specification.
\end{abstract}

\section{Introduction}

The W3C XML Encryption specification [8] is a part of the XML Security standards. It is responsible for ensuring confidentiality in XML-based messages. It is adopted in various standardized scenarios (e.g. Web Services or Single Sign-On) and customized applications. It is a applied in major major web-based applications, ranging from business and e-commerce, financial services and healthcare, to governmental and military infrastructures. It is e.g. implemented in frameworks of several major organizations like Apache, redhat, IBM, Microsoft, SAP.

However, as previously shown [12], the XML Encryption specification is vulnerable to a specific chosen-ciphertext attack. This attack can be applied in all the scenarios, where the attacker is able to send messages including modified ciphertext to an oracle that decrypts the message and responds with 1 or 0 according to the message validity. According to the server responses, the attacker can decrypt the whole ciphertext. The attack is based on a fact that the server parses the decrypted XML data. If the parsing fails, the server interrupts the message processing. This new side-channel has led to a generalization of padding oracle attacks applied by Vaudanay [21] and considered in further works [1, 19, 7 , $22,5,6]$, and to a development of a more performant practical attack. On average the attacker has to issue only 14 requests for decrypting of one byte.

In this paper we summarize the attack and introduce two exemplary scenarios: Web Services and Single Sign-On. We have to note that this attack is however applicable to all the custom applications using XML Encryption and revealing 
a decryption oracle. We summarize discussions with various developers and introduce the proposed countermeasures. We explain why standard-conformant and customized countermeasures in most of the scenarios do not work. We e.g. show why unifying of error messages is not a valid countermeasure or why XML Signatures [9] in Web Services cannot mitigate these attacks if the Web Services are secured using WS-Security Policy [15].

To this end, we propose the standardization of mode of operation including authentication and integrity check. We hope that the discussed difficulties by application of different countermeasures in different scenarios will force the standardization groups to include authenticated modes of operations in their specifications and reconsider deprecation and removing unauthenticated modes of operations $[14,8]$.

\section{Basics}

In this section we give an overview of the Cipher block chaining mode of operation and security standards used in XML and Web Services.

\subsection{Cipher Block Chaining}

AES and 3DES are well-used symmetric-key encryption algorithms. They allow to encrypt and decrypt data, whose length is 128 or 64 bit, respectively. In order to apply these algorithms to data of arbitrary length, the data has to be padded and split into blocks, which are then chained using a mode-of-operation.

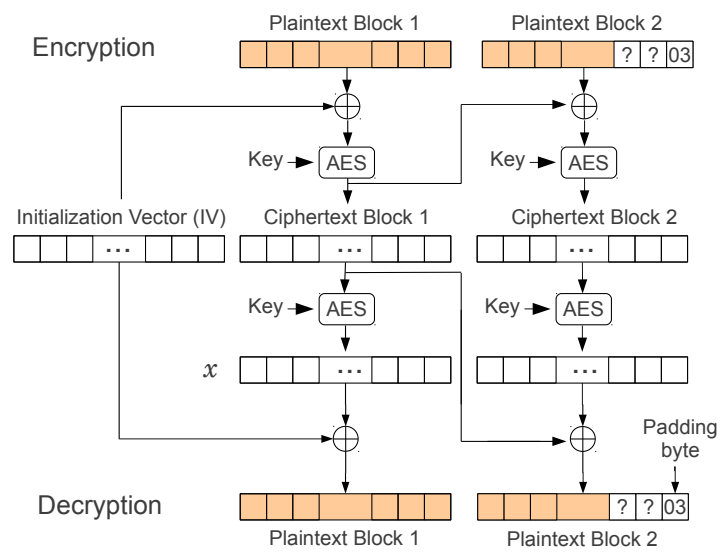

Fig. 1. CBC mode of operation with the XML Encryption padding scheme

Cipher block chaining (CBC) [17] is the most popular blockcipher modeof-operation in practice and the only mode specified in the XML Encryption 
standard. Its functionality in this standard including the padding scheme is depicted in Figure 1.

For its description, suppose a byte string $d a t a^{\prime}$ of arbitrary length. The data string is first padded in order to achieve a length $l$, which is an integer multiple of the block-size bs. XML Encryption specifies the following padding scheme:

1. Compute the smallest integer $p>0$ such that $\left|d a t a^{\prime}\right|+p$ is an integer mutliple of $b s$ of the block cipher.

2. Append $(p-1)$ random bytes to data'.

3. Append one more bytes to data', whose integer value equals $p$.

Using this padding scheme we get data, whose length is multiple of $b s$. Now, we can split data into blocks: data $=\left(\right.$ data $^{(1)}, \ldots$, data $\left.^{(d)}\right)$. These blocks are processed as follows:

- An initialization vector $i v \in\{0,1\}^{8 \cdot b s}$ is chosen at random. The first ciphertext block is computed as

$$
x^{(1)}:=\operatorname{data}^{(1)} \oplus i v, \quad C^{(1)}:=\operatorname{Enc}(k, x) .
$$

- The subsequent ciphertext blocks $C^{(2)}, \ldots, C^{(d)}$ are computed as

$$
x^{(i)}:=\operatorname{data}^{(i)} \oplus C^{(i-1)}, \quad C^{(i)}:=\operatorname{Enc}(k, x)
$$

for $i=2, \ldots, d$.

- The resulting ciphertext is $C=\left(i v, C^{(1)}, \ldots, C^{(d)}\right)$.

The decryption procedure reverts this process in the obvious way.

\subsection{XML and Web Services}

The Extensible Markup Language [2] defines a structure for flexible storage and transmission of tree-based data. It is currently used in Single-Sign On scenarios or in Web Services [11].

Web Services is a W3C standard used to achieve interprocess interactions over networks between different software applications. The communicating applications use SOAP messages [10]. SOAP messages are XML-based messages generally consisting of header and body. The header element includes messagespecific data (e.g. timestamp, user information, or security tokens). The body element contains function invocation and response data, which are mainly addressed to the business logic processors.

\subsection{XML Security}

XML documents often contain data whose confidentiality, authenticity, and integrity must be protected. Therefore, the W3C consortium developed standards describing the XML syntax for applying cryptographic primitives to XML data: XML Encryption [8] and XML Signature [9]. 
XML Encryption XML Encryption specifies a method for achieving confidentiality of the stored XML elements. In order to encrypt XML data, in most scenarios hybrid encryption is used: First, the encryptor chooses a session key $k$. This key is encrypted using a public-key encryption scheme (please note that the public-key encryption scheme is out of scope of this paper since we describe an attack on the part encrypted with a symmetric key). Afterwards, the payload data is encrypted with a symmetric cipher. For this purpose, the standard allows to choose between two symmetric ciphers, namely AES-CBC and 3DES-CBC.

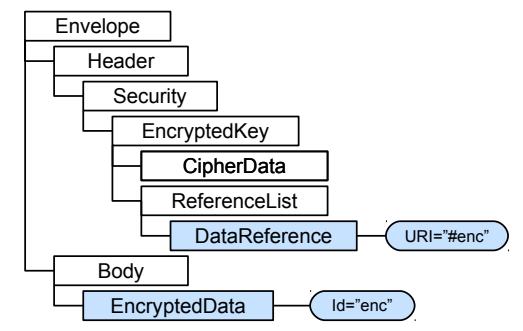

Fig. 2. Example of a SOAP message with encrypted data

Figure 2 gives an example of a SOAP message containing such a hybrid ciphertext. This message consists of the following parts.

1. The EncryptedKey part. This part consists of two components. The CipherData element contains the encrypted session key. Referencelist contains references to all EncryptedData elements that can be decrypted with the session key $k$.

2. The EncryptedData part. This part contains the payload data, encrypted using the session key $k$.

There may be multiple EncryptedData elements sharing the same session key. These elements are referenced using the ReferenceList element.

A SOAP Web Service receiving such an XML document processes it as follows. It first decrypts the session key $k$. Then, it uses $k$ to decrypt all the parts containing encrypted payload. Finally, the payload data is parsed with an XML parser and the whole document is forwarded to the next module or business process invocation.

It is important to mention that the XML Security processing module typically does not know, which parts of the decrypted payload is later processed by the business logic. Thus, it could also happen that the encrypted payload is decrypted, parsed, and not processed further.

XML Signature In order to ensure integrity and authenticity of exchanged XML documents, the XML Security working group defined the XML Signature 




Fig. 3. Example of XML Signature applied on the SOAP body

specification [8]. A simplified structure of an XML Signature applied to a SOAP message according to the WS-Security standard gives Figure 3.

The depicted SOAP message includes a function invocation "MonitorInstances" defined in the SOAP body. The authenticity and integrity of the SOAP body is ensured by the XML Signature defined in the SOAP header. The XML Signature element consists of two mandatory elements: SignedInfo and SignatureValue. The SignedInfo element includes an Id-reference pointing to the SOAP body and a digest value computed over the referenced element. In order to secure the SignedInfo element, a signature value over this element is computed and put to the SignatureValue element. This is achieved by a public-key algorithm such as RSA or DSA.

Processing of the given SOAP message would usually look as follows: The recipient first searches for the referenced element given in SignedInfo. He computes the digest value over this element and compares it to the value given in the DigestValue element. Afterwards, he verifies the signature value over SignedInfo. At the end, he can execute the function defined in the SOAP body.

\subsection{XML Signature Wrapping}

SOAP messages (and other XML documents) containing XML Security are typically processed in two independent steps: signature verification and function invocation (business logic). This observation led to the development of a new attack called XML Signature Wrapping [16]. This attack compromises integrity of the exchanged SOAP messages. An example of this kind of attack applied on the message given in Figure 3 shows Figure 4. In this example an attacker, who is able to eavesdrop the SOAP message, moves the original SOAP body to a Wrapper element in the SOAP header. Afterwards, he creates a SOAP body with a new Id="attack" and defines an arbitrary function, e.g. CreateKeyPair. As the Id of the original SOAP body stays same and the concerning parts are not altered, the security logic can verify its integrity and authenticity. The business logic however invokes the newly inserted function CreateKeyPair.

Let us mention that there exist more sophisticated XML Signature Wrapping attack techniques. They misuse different properties of the validation logic [20] or completely different referencing mechanisms [13]. 


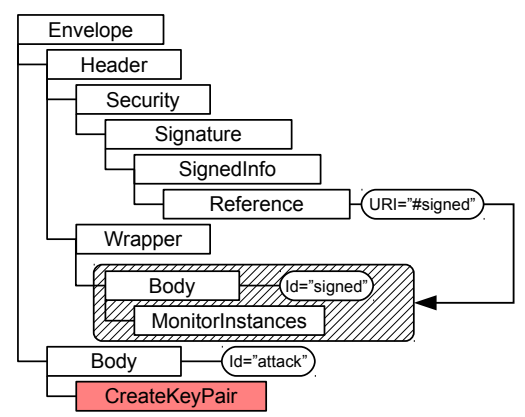

Fig. 4. Signature Wrapping attack

\subsection{WS-Security}

WS-Security [18] is a standard specifying security adoption to Web Services. It specifies how to apply XML Signatures, timestamps, or different tokens in the transmitted SOAP messages. In order to define security properties of the exchanged SOAP messages, both on the server and client side, WS-Security Policy [15] is used. This specification allows to indicate policy assertions that apply to Web Services.

Policy assertions are grouped into policy alternatives. A set of policy alternatives gives a WS-Policy definition. For grouping of policy assertions two XML tags are used: All and ExactlyOne. All indicates that all child node assertions have to be fulfilled. ExactlyOne indicates the logical OR and it contains assertions, from which at least one has to be fulfilled.

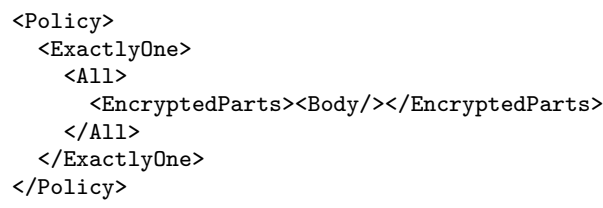

Fig. 5. WS-Security Policy defining that the SOAP body in the incoming message has to be encrypted. However, it does not restrict encryption of other elements.

An example of WS-Security Policy document gives Figure 5. This Policy definition would enforce the server to process only the SOAP messages that contain encrypted SOAP body. It is however important to mention that using this policy a server would also accept SOAP messages including additional encrypted document parts, e.g. elements in the SOAP header. 


\subsection{Single Sign-On and SAML}

The growing number of user identities in Internet has led to development of different Single Sign-On solutions. These solutions are based on one login-procedure. The users authenticate only by a trustworthy Identity Provider (IP). After successful login, Identity Provider can issue different tokens for the user giving him access to Relying Parties (RP). Single Sign-On can be realized by different standards including OAuth, OpenID, or Security Assertion Markup Language (SAML) [4].

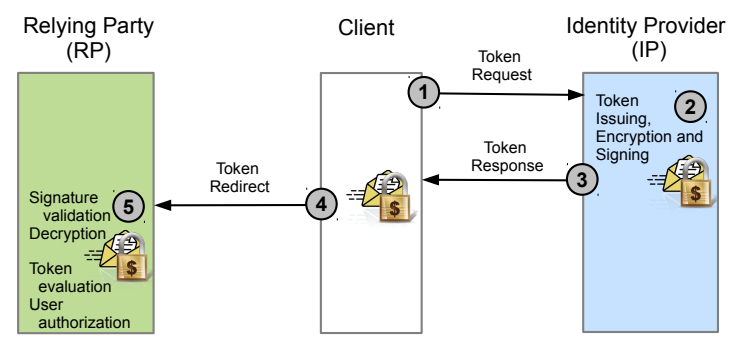

Fig. 6. A typical SAML-based Single Sign-On scenario: The user sends a token request to IP. IP issues a token for the user, which is afterwards encrypted and signed. The issued token is sent to the user and forwarded to RP.

In the following, we will consider the XML-based SAML standard. This standard allows to define arbitrary claims about the identities and secure them by applying XML Signature and XML Encryption. Please note that the structure of SAML-based messages is out of scope of this paper. It is only important to know that the confidentiality of these messages is secured using the XML Encryption specification.

A simplified SAML-based Single Sign-On scenario is depicted in Figure 6. In this scenario, a logged-in user first sends a token request including the desired RP to IP (1). IP issues a token for the user including e.g. his rights, roles, or expiration time. The token is afterwards encrypted and signed (2). IP sends the secured token to the user (3), who forwards it to the desired RP (4). RP first validates the signature over the token and decrypts it. Afterwards, it evaluates the token and gives the user rights to access his domain (5).

\section{High-Level Attack Description}

In this section we give a brief attack description. Afterwards, we show two realworld attack scenarios. Please note that these scenarios are only exemplary, the attack can also be applied to other custom applications. 


\subsection{Attack Description}

The attack on XML Encryption is based on the malleability of the CBC mode of operation: It allows an attacker, who is in possession of the ciphertext $C=$ $\left(i v, C^{(1)}\right)$, to flip arbitrary bits in the plaintext $m=\operatorname{Dec}(C, k)$ without knowing the session key $k$. He can simply achieve this by flipping appropriate bits in the initialization vector $i v$.

Changing different bits in the encrypted XML data can lead to errors in the server processing, which forces the server to respond with a fault message. These fault messages can have the following reasons:

- Decryption errors. This error stems from an incorrect padding. Recall that the last byte of a padded plaintext must include a valid padding number.

- Parsing errors. This error may have two reasons. Either the plaintext contains an "unprintable" ASCII character (XML parser can parse only valid characters, otherwise it stops processing). The other reason is that the syntax of the decrypted XML part is not valid. The latter means that the special escape character $0 x 38(\&)$ is not followed by a valid entity reference, or the bracket $0 x 60(<)$ is not properly closed.

Sending differently adapted ciphertexts to the server and observing the server responses gives the attacker the possibility to efficiently decrypt the eavesdropped ciphertext.



Fig. 7. An example of the attack on the ciphertext block containing the incomplete plaintext <expires>2011</ 
Given a ciphertext $C=\left(C^{0}, C^{1}, \ldots, C^{d}\right)$, which contains valid XML data including no escape characters $(\&)$, the algorithm looks as follows. The attacker performs the attack in $d$ rounds (each ciphertext block is decrypted in each different round). In each round $i=1 \ldots d$, he takes two ciphertext blocks $c=$ $\left(C^{i-1}, C^{i}\right)=\left(I V, C^{i}\right)$ and with these two blocks he proceeds as follows:

1. He excludes all the left brackets from the plaintext in order to overcome the parsing errors originating from improperly closed elements. To this end, he iterates over the last $I V$ byte and sends in each iteration the two blocks $c=\left(I V^{\prime}, C^{i}\right)$ to the server. The number of valid responses gives him the position of the first left bracket. Afterwards, he can flip the bit in the byte containing ' $<$ to convert it to a different valid character. He repeats this step until he excludes all the brackets.

2. He sets the last $I V$ byte so that the last plaintext byte contains $0 x 01$. This gives him a possibility to access all the proceeding bytes in the block.

3. Now, he is able to decrypt all the bytes in the block byte-by-byte. Thereby, he uses server error responses returned by invalid parsing processing.

An example of processing these three steps is depicted in Figure 7.

After execution of these three steps, the attacker has knowledge of vector $x^{(i)}=d a t a^{(i)} \oplus i v$ (see Section 2.1). Therefore, he is also able to encrypt arbitrary XML elements.

\subsection{Attack Scenario: Web Services}

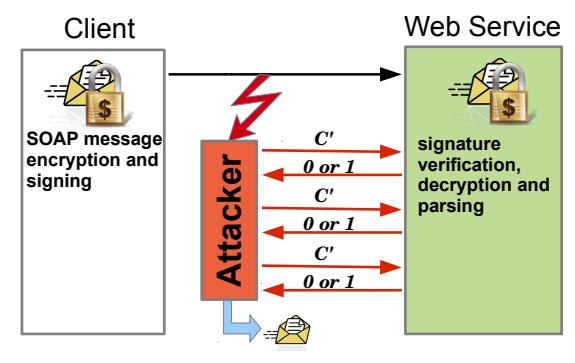

Fig. 8. Attack on Web Services: Adversary sends eavesdropped SOAP message with modified ciphertexts $c^{\prime}$ to the Web Service server and observes the server responses. With each response he learns a few plaintext bits.

The first scenario describes an attack on a Web Service server and is depicted in Figure 8. In this scenario the adversary first eavesdrops a SOAP message transmitted between the client and the Web Service server. He modifies the ciphertext $c$ included in the SOAP message according to the algorithm described above. He sends the message to the server and observes its response. He needs only to distinguish between two response types: fault (1) and valid (0). According 
to the response type he is able to learn a few bits of the plaintext and construct new ciphertexts $c^{\prime}$. He repeats this step until he decrypts the whole encrypted content.

Please note that the XML Signatures in SOAP messages are of no help. We explain this later in the next section.

\subsection{Attack Scenario: SAML-based Single Sign-On}

The second scenario presents an attack applied in SAML-based Single Sign-On and is depicted in Figure 9. It can be executed by an adversary who issues modified ciphertexts, includes them into the SAML assertions, and sends them to RP. If RP responds with 1 or 0 according to the message validity, the adversary can distinguish between valid and invalid ciphertexts. This gives him the possibility to decrypt the whole assertion or create new ciphertexts with arbitrary content.

In this scenario we have to consider also an adversary who is registered by IP and is a valid user of RP. By applying the attack, this adversary can modify the encrypted SAML assertion sent from IP in order to execute privilege escalation and e.g. get administrator rights. Please note that in this case SSL/TLS would be of no help since the adversary is able to see the forwarded SAML messages.



Fig. 9. Attack in a SAML-based scenario: the adversary gains the encrypted SAML message. He applies the attack by sending the modified SAML assertions to RP.

\section{Countermeasures}

In this section we give an overview of some countermeasures against the attack on XML Encryption and we analyze the scenarios, in which they work.

\subsection{XML Signature}

Application of XML Signatures on ciphertexts can ensure their authenticity and integrity. This standard describes two types of signatures, namely public-key 
XML Signatures (which use classical digital signature schemes) and secret-key XML Signatures (which use message authentication codes).

Generally, XML Signatures can thwart these attacks if and only if:

1. the attacker is not able to create validly signed messages.

2. the encrypted part cannot be moved to any unsigned part of the document.

If the application could ensure these two points, the attack could not be applied. However, in the following we illustrate that this is not that trivial. For this purpose, please consider the SOAP message depicted in Figure 10. In this message the SOAP body contains an encrypted payload, which is signed using XML Signature and Id-based referencing.



Fig. 10. XML Signature applied on the encrypted payload in a SOAP message

Attacker Able to Create Validly Signed Messages First problem by application of public-key XML Signatures comes with a scenario where more parties are allowed to communicate with a Web Service server. Consider for instance there are two clients $E_{1}$ and $E_{2}$ of a Web Service provider, where both clients can send encrypted and digitally signed messages to the server. Assume that $E_{1}$ creates a SOAP message with encrypted and signed content, and sends it to the server. Now if $E_{2}$ wants to learn the contents of this message, then it could record this message, simply remove the signature, compute its own signature over the ciphertext, and mount the attack. The crucial point here is that the server cannot distinguish whether $E_{2}$ has encrypted the payload itself, or copied it from a ciphertext that $E_{1}$ has created. Still, in the digital signature setting the server can at least identify the attacker uniquely.

XML Signature Wrapping Another problem with application of XML Signatures on ciphertexts is XML Signature Wrapping attack. This attack affects also secret-key XML Signatures.

Figure 11 gives an example of signature wrapping attack application on the message presented in the previous figure. In order to execute this attack, the attacker first copies the authenticated SOAP body into the security header. As 
the Id of the SOAP body stays the same, the signature component is able to validate this element. Afterwards, the attacker needs only to apply the attack on XML Encryption on the content of the newly defined SOAP body: he must force the server to decrypt the content of this element. Thus, he simply changes the DataReference element in EncryptedKey and makes it point to the content of the newly defined SOAP body.



Fig. 11. XML Signature wrapping moves the signature validation to the SOAP header and thereby offers a new possibility for mounting of the XML Enryption attack

The server would process the depicted message as follows. It would first validate the XML Signature. Afterwards, it would decrypt and parse the content of the newly defined SOAP body. After successful data decryption and parsing, the payload would be processed by the business logic. This step would most probably fail, since the randomly generated payload could not be processed by the server's business logic. Thus, by applying this attack the attacker must rely on differences between fault messages coming from the decryption processing and the business logic.

We evaluated Apache Axis2 and JBossWS regarding their resistance against XML Signature Wrapping attacks. Our experiments showed that all basic examples are vulnerable to simple XML Signature wrapping attacks. Thus, the attacker could mount the the attack on XML Encryption.

Similar attacks can be executed on SAML, where the signed elements can be moved to various unsigned parts and the decryption of unsigned elements can be enforced. The concrete description is however out of scope of this paper.

XML Encryption Wrapping One could say signature wrapping attacks could be thwart using different countermeasures. Apache Axis2 e.g. uses WS-Security Policy verification, which explicitly checks, if the SOAP body is signed (this is a generally applicable countermeasure). This countermeasure makes it impossible to move the signed SOAP body to another parts of the document. However, as described in Section 2.5, WS-Security Policy cannot define the maximal number 
of secured elements. This gives the attacker an opportunity to force the server to decrypt additional elements and apply the so called XML Encryption Wrapping attack.

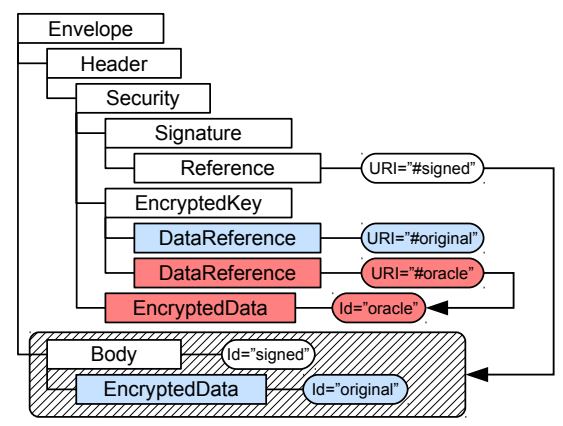

Fig. 12. XML Encryption wrapping copies the encrypted payload to an unsigned document part and thereby offers the attacker construction of a server oracle

The basic idea of this attack is depicted in Figure 12. By its application the attacker simply copies the encrypted payload to any unsigned document part, which does not violate XML Schema. Afterwards, the attacker creates a new DataReference inside of the EncryptedKey element and makes it point the newly created EncryptedData element (please note that the attacker can also create a completely new EncryptedKey element). This forces the server to decrypt both EncryptedData elements. The original encrypted payload stays in the signed part. Thus, the XML Signature stays valid, the original payload is decrypted and parsed, and the server's business logic processes it without failures. On the other hand, the newly created EncryptedData element in the SOAP header is decrypted and parsed. The business logic does not process it, as it only needs the content of the SOAP body.

By application of this simple attack the attacker creates a server oracle, which responds with 0 or 1 depending on the validity of the ciphertext. Therefore, even encrypted server responses could simply be recognized and the attack applied.

We tested all Rampart and JBoss configuration examples, showing their vulnerability against XML Encryption wrapping.

\subsection{Unifying of Error Messages}

The possibly most obvious countermeasure to our attack consists in unifying the SOAP fault messages sent in response to invalid SOAP request messages so that an attacker can not distinguish between a decryption error and an application level error. However, this approach has some serious drawbacks.

At first, meaningful error messages are generally considered as "good programming practice". In fact, they are necessary for developers that have to implement client-side applications for encryption-enabled Web Services. 
Secondly, even with unified SOAP fault messages, there are additional sidechannels that can be exploited for determining what type of error a certain request message triggered. For instance, measuring the time consumed until a (unified) SOAP fault message arrives may already indicate the level in the application stack at which the error occurred.

Finally, we stress that this countermeasure is not effective when XML Encryption wrapping attacks as described above are applicable, since copying the encrypted data to a deeper level in the SOAP header would exclude them from XML Schema validation and business logic processing. Thus, the server would respond with a SOAP fault if and only if the encrypted data in the SOAP header are incorrect.

\subsection{Another Countermeasures}

In this section we describe other countermeasures, which were proposed by different software vendors.

Revocation of Session Keys By application of the attack on one EncryptedData element the attacker uses for each server request the same symmetric key. Revocation of symmetric keys could be considered as a valid countermeasure. However, this countermeasure could cause the following problems:

- It needs to apply serious changes to applications or libraries.

- It requires a shared state across servers that are working in a cluster. Even if this state would be achieved, it would potentially be possible to get some responses back before all the servers know about the revoked key.

- Sometimes, even a few bits of information are enough to decrypt an important part of a message. You can e.g. think of messages including boolean values ("yes"/"no") or credit card numbers.

Blacklisting of Clients' Public Keys This countermeasure would bring similar problems as described above. However, it would partially solve the publickey signature problems described in Section 4.1 as the not honest clients having server access would be blocked after sending invalid messages.

Inclusion of Signed Nonces Inclusion of signed nonces could be seen as another valid countermeasure. However, it causes similar problems as the above mentioned revocation of session keys. Moreover, its application includes signature problems as described in Section 4.1.

Blacklisting of Clients' IP addresses A countermeasure proposed by some developers is blocking the clients that already sent a few number of invalid messages causing security faults. Please note that there are the same drawbacks as in the two countermeasures described above. Moreover, this countermeasure 
does not work if the attacker is able to execute his attack from machines with different IP addresses. This is a valid assumption when considering allocation of virtual instances in cloud scenarios.

Another drawback would be a simple application of Denial-of-Service attacks. The attacker having the same IP as the honest client could send an amount of invalid messages causing security errors. This would again be a valid assumption when considering that attacker and honest client stay in the same subnet and are accessing a remote server.

Decryption only of Signed Elements As described in the previous sections, the WS-Policy unfortunately does not allow to restrict element decryption. This makes it possible to apply XML Encryption wrapping attacks. Moreover, it is possible to execute Denial-of-Service attacks by inclusion of a large number of EncryptedKey elements, which force the server to execute expensive publickey decryption. Therefore, we propose inclusion of new policy tags allowing to decrypt the element only if it is signed.

Please note that application of this countermeasure still would not solve signature problems as described in Section 4.1.

\subsection{Changing Mode of Operation}

Finally we would like to highlight some cryptographic countermeasures. Unfortunately, all proposals require to modify the current version of the XML Encryption standard.

One option is to use a symmetric cryptographic primitive that does not only provide confidentiality, but also integrity. One option may be to add a message authentication code (MAC) like HMAC (see [17]) over the ciphertext to the encrypted message. In contrast to a digital signature, which can simply be replaced by a different signature, the security properties of a MAC ensure that it is not be possible for an attacker to modify a ciphertext while keeping the MAC valid. In this case, our attack becomes impossible. Another option, which provides the same improvement in security, would be to replace the CBC mode of operation with a mode of operation that provides message integrity, like the Galois counter mode (GCM) [17], for instance.

Streaming-based XML Encryption processing Even when applying message authentication codes, the developers should pay attention to other sidechannel attacks. These can appear when applying streaming-based XML processing.

Consider an XML security module that accepts an encrypted byte stream. It decrypts this stream and sends it block-by-block to a streaming-based parser. The parser processes the incoming elements and sends them to another processing module. At the end of the stream the security module checks the MAC over all encrypted data. Consider that if a decryption or parsing error occurs, the 
parser interrupts the message processing and immediately sends a fault message. Moreover, consider an attacker who is in possession of a valid encrypted text and is able to send it to the server with different IVs (this is also possible when applying GCM). If the encrypted text is long enough, the attacker could send messages with different IVs and observe the server response time. Longer response time would indicate correct payload and failure by the MAC verification. Shorter response time would indicate an incorrect payload.

This is a valid assumption also when applying standard DOM-based (treebased) parsers [3]. Namely, some DOM-parsers include an underlying streamingparser, which is used for preprocessing of the incoming elements. Therefore, developers should pay attention when implementing modes of operations including MAC-checking: The encrypted part should always be completely processed and parsed and the MAC should be validated afterwards. Otherwise, different sidechannels could appear.

\section{Acknowledgments}

We would like to thank Thomas Alt, Martijn de Boer, Scott Cantor, Florian Kohlar, Tibor Jager, Jan Lieskovsky, Martin Rex, and Alessio Soldano for their contributions.

\section{References}

1. John Black and Hector Urtubia. Side-channel attacks on symmetric encryption schemes: The case for authenticated encryption. In Dan Boneh, editor, USENIX Security Symposium, pages 327-338. USENIX, 2002.

2. Tim Bray, Jean Paoli, C. M. Sperberg-McQueen, Eve Maler, and François Yergeau. Extensible Markup Language (XML) 1.0 (Fifth Edition). W3C Recommendation, 2008.

3. Steve Byrne, Arnaud Le Hors, Philippe Le Hégaret, Mike Champion, Gavin Nicol, Jonathan Robie, and Lauren Wood. Document object model (DOM) level 3 core specification. W3C recommendation, W3C, April 2004. http://www.w3.org/TR/ 2004/REC-DOM-Level-3-Core-20040407.

4. Scott Cantor, John Kemp, Rob Philpott, and Eve Maler. Assertions and Protocol for the OASIS Security Assertion Markup Language (SAML) V2.0. OASIS Standard, 15.03.2005, 2005. http://docs.oasis-open.org/security/saml/v2. 0/saml-core-2.0-os.pdf.

5. Jean Paul Degabriele and Kenneth G. Paterson. Attacking the IPsec standards in encryption-only configurations. In IEEE Symposium on Security and Privacy, pages 335-349. IEEE Computer Society, 2007.

6. Jean Paul Degabriele and Kenneth G. Paterson. On the (in)security of IPsec in MAC-then-encrypt configurations. In Ehab Al-Shaer, Angelos D. Keromytis, and Vitaly Shmatikov, editors, ACM Conference on Computer and Communications Security, pages 493-504. ACM, 2010.

7. Thai Duong and Juliano Rizzo. Cryptography in the web: The case of cryptographic design flaws in ASP.NET. In IEEE Symposium on Security and Privacy, 2011. 
8. Donald Eastlake, Joseph Reagle, Takeshi Imamura, Blair Dillaway, and Ed Simon. XML Encryption Syntax and Processing. W3C Recommendation, 2002.

9. Donald Eastlake, Joseph Reagle, David Solo, Frederick Hirsch, and Thomas Roessler. XML Signature Syntax and Processing (Second Edition). W3C Recommendation, 2008.

10. Martin Gudgin, Marc Hadley, Noah Mendelsohn, Jean-Jacques Moreau, and Henrik Frystyk Nielsen. SOAP Version 1.2 Part 1: Messaging Framework. W3C Recommendation, 2003.

11. Hugo Haas, David Booth, Eric Newcomer, Mike Champion, David Orchard, Christopher Ferris, and Francis McCabe. Web services architecture. W3C note, W3C, February 2004. http://www.w3.org/TR/2004/NOTE-ws-arch-20040211/.

12. Tibor Jager and Juraj Somorovsky. How To Break XML Encryption. In The 18th ACM Conference on Computer and Communications Security (CCS), October 2011.

13. Meiko Jensen, Lijun Liao, and Jörg Schwenk. The curse of namespaces in the domain of XML signature. In ACM Workshop on Secure Web Services (SWS), pages 29-36, 2009.

14. M. Jones, E. Rescorla, and J. Hildebrand. Json web encryption (jwe) draft-jones-json-web-encryption-01, oct 2011. http://tools.ietf.org/html/ draft-jones-json-web-encryption-01.

15. Chris Kaler and Anthony Nadalin. Web Services Security Policy Language (WSSecurityPolicy) 1.1. 2005.

16. Michael McIntosh and Paula Austel. XML signature element wrapping attacks and countermeasures. In SWS '05: Proceedings of the 2005 workshop on Secure web services, pages 20-27, New York, NY, USA, 2005. ACM Press.

17. Alfred J. Menezes, Paul C. van Oorschot, and Scott A. Vanstone. Handbook of Applied Cryptography. CRC Press, Boca Raton, Florida, 1996.

18. Anthony Nadalin, Chris Kaler, Ronald Monzillo, and Phillip Hallam-Baker. Web Services Security: SOAP Message Security 1.1 (WS-Security 2004). OASIS Standard, 2006.

19. Kenneth G. Paterson and Arnold Yau. Padding oracle attacks on the ISO CBC mode encryption standard. In Tatsuaki Okamoto, editor, Topics in Cryptology CT-RSA 2004, volume 2964 of Lecture Notes in Computer Science, pages 305-323. Springer, February 2004.

20. Juraj Somorovsky, Mario Heiderich, Meiko Jensen, Jörg Schwenk, Nils Gruschka, and Luigi Lo Iacono. All Your Clouds are Belong to us - Security Analysis of Cloud Management Interfaces. In The ACM Cloud Computing Security Workshop (CCSW), October 2011.

21. Serge Vaudenay. Security flaws induced by CBC padding - applications to SSL, IPSEC, WTLS ... In Lars R. Knudsen, editor, Advances in Cryptology - EUROCRYPT 2002, volume 2332 of Lecture Notes in Computer Science, pages 534-546. Springer, April / May 2002.

22. Arnold K. L. Yau, Kenneth G. Paterson, and Chris J. Mitchell. Padding oracle attacks on CBC-mode encryption with secret and random IVs. In Henri Gilbert and Helena Handschuh, editors, Fast Software Encryption - FSE 2005, volume 3557 of Lecture Notes in Computer Science, pages 299-319. Springer, February 2005. 